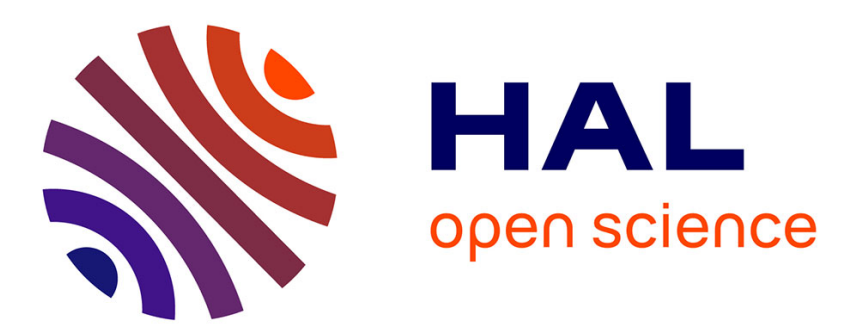

\title{
Infection dynamics of Nosema ceranae in honey bee midgut and host cell apoptosis
}

Christoph Kurze, Yves Le Conte, Per Kryger, Oleg Lewkowski, Thomas Müller, Robin F.A. Moritz

\section{- To cite this version:}

Christoph Kurze, Yves Le Conte, Per Kryger, Oleg Lewkowski, Thomas Müller, et al.. Infection dynamics of Nosema ceranae in honey bee midgut and host cell apoptosis. Journal of Invertebrate Pathology, 2018, 154, pp.1-4. 10.1016/j.jip.2018.03.008 . hal-02624305

\section{HAL Id: hal-02624305 \\ https: / hal.inrae.fr/hal-02624305}

Submitted on 26 May 2020

HAL is a multi-disciplinary open access archive for the deposit and dissemination of scientific research documents, whether they are published or not. The documents may come from teaching and research institutions in France or abroad, or from public or private research centers.
L'archive ouverte pluridisciplinaire HAL, est destinée au dépôt et à la diffusion de documents scientifiques de niveau recherche, publiés ou non, émanant des établissements d'enseignement et de recherche français ou étrangers, des laboratoires publics ou privés. 
Short Communication

\title{
Infection dynamics of Nosema ceranae in honey bee midgut and host cell apoptosis
}

\author{
Christoph Kurze ${ }^{\mathrm{a}, \mathrm{b}, *}$, Yves Le Conte ${ }^{\mathrm{c}}$, Per Kryger ${ }^{\mathrm{d}}$, Oleg Lewkowski ${ }^{\mathrm{a}}$, Thomas Müller ${ }^{\mathrm{e}}$, \\ Robin F.A. Moritz ${ }^{\mathrm{a}, \mathrm{f}, \mathrm{g}}$

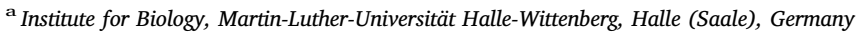 \\ ${ }^{\mathrm{b}}$ Department of Entomology and Center for Infectious Disease Dynamics, Pennsylvania State University, University Park, PA 16802, United States \\ c INRA, UR 406 Abeilles et Environnement, Avignon, France \\ ${ }^{\mathrm{d}}$ Department of Agroecology, Aarhus University, Flakkebjerg, Denmark \\ e Department of Internal Medicine IV, Martin-Luther-Universität Halle-Wittenberg, Halle (Saale), Germany \\ ${ }^{\mathrm{f}}$ University of Pretoria, Department of Zoology and Entomology, Pretoria, South Africa \\ ${ }^{\mathrm{g}}$ University of Veterinary and Agricultural Sciences, Cluj-Napoca, Romania
}

\begin{abstract}
A B S T R A C T
Nosema ceranae is an intracellular microsporidian parasite that infects epithelial cells of the honey bee (Apis mellifera) midgut. Previous studies have shown that Nosema may alter cell renewal and apoptosis in honey bees. We found that the amount of apoptotic cells progressively declines from the anterior towards posterior regions of the midgut in Nosema-infected sensitive bees. There was no such pattern in the infected Nosema tolerant honey bees and controls. These data provide additional evidence that $N$. ceranae appears to alter apoptosis in its host cells for its own advantage.
\end{abstract}

\section{Introduction}

The microsporidian parasite Nosema ceranae is a globally emerging pathogen that infects honey bees (Apis mellifera) (Klee et al., 2007). Hence, the effects of $N$. ceranae on its host and host immune defenses have been studied in considerable detail over the last decade (Fries et al., 2013; Higes et al., 2013b; Kurze et al., 2016c). Nosema is primarily transmitted via the fecal-oral route between adult bees and establishes an infection in the midgut epithelium (Fries, 2010). Over the course of infection, N. ceranae has been shown to induce multiple physiological alterations in its host, including processes involved in the regulation of programmed cell death (PCD) (Doublet et al., 2017; Higes et al., 2013a; Kurze et al., 2015; Martin-Hernandez et al., 2017) and energy metabolism (Kurze et al., 2016b; Mayack and Naug, 2009; Vidau et al., 2014). This might be not surprising as microsporidia rely on their host metabolism for supply of energy resources (Keeling, 2009). A breeding program selecting against Nosema over 20 years resulted in a Nosema tolerant honey bee lineage (Hatjina et al., 2014). Although Nosema prevalence reduced from $60-80 \%$ down to about $10 \%$ in selected colonies (Hatjina et al., 2014), individual bees were still susceptible to both Nosema apis and $N$. ceranae in laboratory experiments (Kurze et al., 2016b). Interestingly, Huang et al. (2012) showed a higher survival and increased immune responses in those tolerant bees compared to sensitive bees from an unselected lineage, despite suffering similar infection intensities of $N$. ceranae. In subsequent experiments, infected worker bees from this Nosema tolerant linage appeared to neither experience decreased rates of apoptosis (one form of PCD) (Kurze et al., 2015) nor energetic stress (Kurze et al., 2016b). A similar pattern was also found when comparing the midgut proteomes between infected workers of both the Nosema tolerant and a Nosema sensitive lineage (Kurze et al., 2016a).

Although the midgut is clearly the major site of the interaction between Nosema and honey bee, specific data on the actual dynamics along the host's midgut epithelium are lacking. In this study, we focused on patterns of apoptosis in response to $N$. ceranae infection in different regions of the midgut after six days post infection (dpi). We quantified the rates of apoptosis in longitudinal sections of the midgut in Nosema tolerant and sensitive bees to gain a better understanding of the local effects caused by $N$. ceranae.

\footnotetext{
* Corresponding author at: Department of Entomology and Center for Infectious Disease Dynamics, Pennsylvania State University, University Park, PA 16802, United States.

E-mail address: cuk210@psu.edu (C. Kurze).
} 


\section{Materials and methods}

\subsection{Experimental inoculation}

We conducted three experimental replicates at the Unité de Recherche Abeilles et Environnement de l'INRA Avignon (France) as described previously (Kurze et al., 2015). Briefly, twenty newly emerged workers ( $<24 \mathrm{~h}$ old) from both Nosema sensitive (SN) and Nosema tolerant colonies (TN, originally from an apiary in Aarhus, Denmark) were individually fed with freshly extracted and purified $50,000 N$. ceranae spores $\mu^{-1}$ in $2 \mu$ of $50 \%(w / v)$ sucrose solution. Uninfected controls of the sensitive (SC) and tolerant (TC) strains were fed with $2 \mu \mathrm{l}$ sucrose solution without spores. We did not include bees in the experiments that had not consumed the entire inoculum. Each treatment group (i.e. SC, TC, SN and TN) was separately housed in autoclaved cages $(10 \times 10 \times 5.5 \mathrm{~cm})$ with a piece of clean wax foundation in an incubator at $34 \pm 1{ }^{\circ} \mathrm{C}, 60 \%$ relative humidity and provided with $50 \%(\mathrm{w} / \mathrm{v})$ sucrose solution ad libitum. Experiments were terminated after six days' post-infection (dpi).

\subsection{Spore counts}

We dissected midguts (ventriculi without rectum) for a random subset of 5 bees for each treatment group and cage replicate. Subsequently, midguts were homogenized in $1000 \mu \mathrm{l}$ of distilled water and the numbers of mature spores were counted with a Fuchs-Rosenthal hemocytometer using a phase-contrast microscope at $\times 400$ magnification.

\subsection{Immunohistochemistry}

We randomly chose three bees per treatment group and replicate to dissect their midguts (ventriculi without rectum). These were subsequently fixed in $4 \%$ buffered formaldehyde for $24 \mathrm{~h}$ at $8{ }^{\circ} \mathrm{C}$ and embedded in paraffin according to standard methods. To visualize apoptotic cell at the early stage, we conducted TUNEL (Terminal deoxynucleotide transferase mediated X-dUTP nick endlabelling) assays (In Situ Cell Death Detection Kit, Roche Dianostics GmbH, Mannheim, Germany) on $7 \mu \mathrm{m}$ thick longitudinal sections following manufacturer's recommendations. Prior to the TUNEL reaction, endogenous peroxidase activity was blocked (Dual Endogenous Enzyme Block, Dako Denmark, Glostrup. Denmark), followed by permeabilisation step using nucleases-free $10 \mu \mathrm{gl}^{-1}$ proteinase $\mathrm{K}$ in $10 \mathrm{mM}$ Tris/ $\mathrm{HCl} \mathrm{pH} 7.5$ for $20 \mathrm{~min}$ at room temperature. Then we rinsed samples twice in PBS (phosphate-buffered saline). After an incubation time of $1 \mathrm{~h}$ at $37^{\circ} \mathrm{C}$ in the dark, the TUNEL reaction was stopped by rinsing the samples three times in PBS and follow by counterstaining reaction with $1 \mu \mathrm{g} \mathrm{ml}{ }^{-1}$ DAPI $\left(4^{\prime}, 6-\right.$ diamidino-2-phenylindole) (Sigma-Aldrich, St. Louis, United States). We quantified the relative number of apoptotic cells (TUNEL + ve, Fig. 1) in the anterior, central and posterior regions of the midgut epithelium as described previously (Kurze et al., 2015). Briefly, we compiled four images per region visualizing the TUNEL and DAPI stain (microscope Axiovert25, camera AxioCam, software Axiovision 4.6; all from Zeiss, Jena, Germany) and images were automatically analyzed in Fiji (Schindelin et al., 2012) (Fig. 1). Only samples with undisrupted epithelium from anterior until posterior midgut (i.e. due to microtome sectioning) and good image quality have been included into the analysis. Final samples sizes were $n=9$ (SC), $n=7(\mathrm{SN}), \mathrm{n}=7$ (TC) and $\mathrm{n}=7$ (TN).

\subsection{Statistics}

All statistical analyses and data plotting (mean $\pm 95 \%$ confidence intervals, CI) were carried out in R (v.3.0.2). Spore load between infected groups was tested using Welch's two-tailed $t$-test. To test the effects on the apoptosis ratio, we ran Linear Mixed-Effects Models
(LMMs) using the lmer function of the lme4 package (v.1.1-12). If a model was found to be unstable with all interactions included, we removed non-significant interactions step-wise. Model validity was tested by comparing full models to their null models without any fixed factors included using maximum likelihood ratio test. Additionally, we checked the data for normality and residuals. The final model including the treatment, bee lineage, midgut regions and their interactions as fixed factors and nested them under each individual bee as random factor. We used the glht function of the multcomp package (v.1.3-2.) for between group comparisons, correcting for multiple testing using the 'false discover rate'.

\section{Results and discussion}

The insect midgut is a dynamic organ, which is essential for immune defense, digestion and detoxification. To maintain these functions, senescent, damaged and infected cells are replaced through PCD (including apoptosis) and asymmetric cell division (Lee et al., 2017). In our experiment, we found that one-week old uninfected control bees (SC and TC) maintained overall an average cell turnover of $40 \% \pm 3 \mathrm{CI}$ $(n=16)$ from the anterior to the posterior part of the midgut (Fig. 2). Despite a slight reduction of apoptosis in the posterior midgut (LMM: estimate \pm s.e. $=-0.072 \pm 0.032, \mathrm{p}<0.05$; Fig. 2 ), we found the strongest effect for the interactions among bee lineages, midgut regions and treatments (LMM: $80.197 \pm 0.070, \mathrm{p}<0.01$ ). There was a highly significant difference in apoptosis between the anterior with $40 \% \pm 12$ $\mathrm{CI}$ and posterior region with $22 \% \pm 10 \mathrm{CI} \quad(\mathrm{n}=7)$ in $\mathrm{SN}$ $(0.197 \pm 0.036, p<0.0001$; Fig. 2). The rate of apoptosis was also slightly lower when comparing the anterior with central regions in those bees $(0.097 \pm 0.036, p<0.05$; Fig. 2). Furthermore, apoptosis in the central and posterior midgut were significantly lower in $\mathrm{SN}$ compared to their controls SC $(-0.143 \pm 0.046, \mathrm{p}<0.05$ and $-0.138 \pm 0.046, p<0.05$ respectively; Fig. 2). As infestation appeared to increase from the anterior to the posterior epithelium at this relatively early stage of infection (Fries, 1988), our results strongly support previous suggestions that $N$. ceranae inhibits host cell apoptosis (Higes et al., 2013a; Kurze et al., 2015; Martin-Hernandez et al., 2017). An important role of PCD in bee-Nosema interactions was also indicated by proteomic and transcriptomic studies (Doublet et al., 2017; Dussaubat et al., 2012; Holt et al., 2013; Kurze et al., 2016a).

Although overall there was no difference between the numbers of Nosema spores in sensitive (SN: $6.0 \pm 1.2 \times 10^{6}$ spores; $\mathrm{n}=14$ ) and tolerant honeybees (TN: $8.0 \pm 1.4 \times 10^{6}$ spores; $\left.n=15\right)$ after 6 dpi $(t$ test: $\mathrm{t}=1.115$, d.f. $=26.77, \mathrm{p}>0.05$ ), we did not observe reduced apoptosis along the epithelium in $\mathrm{TN}(36 \% \pm 3 \mathrm{CI}$; $0.019 \pm 0.036$, $\mathrm{p}>0.05$; Fig. 2). Despite severe infection levels, TN maintained similarly highly levels of apoptosis in the posterior region $(38 \% \pm 2 \mathrm{CI}$, $\mathrm{n}=7)$ as control bees $(0.050 \pm 0.049, \mathrm{p}>0.05)$, but their levels were significantly higher as in SN $(0.156 \pm 0.049$, $\mathrm{p}<0.05$; Fig. 2$)$. The high apoptosis levels of midgut epithelium in the Nosema tolerant bee linage might had been suggested to reduce the infection intensity over a longer period through defecation outside the hive and thereby also reducing the risk of horizontal transmission (Kurze et al., 2015).

Since the posterior insect midgut is an important site for virus acquisition (for example in mosquitos and aphids, McGee et al., 2010; Reinbold et al., 2003; Vaidyanathan and Scott, 2006), reduced apoptosis of almost $50 \%$ in SN may facilitate secondary infections that could lead to increased mortality. Although $N$. ceranae has been suggested to promote virus infections by suppressing the host immune system (Antúnez et al., 2009), it has been recently shown to have also negative effects on viruses (Doublet et al., 2015). The study of any interactions between Nosema with other agents in the gut microbiome is therefore clearly a rewarding topic for future research. Nevertheless, the regiondependent dynamics of the interactions between $N$. ceranae and the host cell epithelium, are an important step towards understanding the pathophysiology of Nosema infections. 
Version définitive du manuscrit publiée dans / Final version of the manuscript published in : Journal of Invertebrate Pathology (2018), Vol. 154, p. 1-4, DOI: 10.1016/j.jip.2018.03.008 Journal homepage: www.elsevier.com/locate/jip
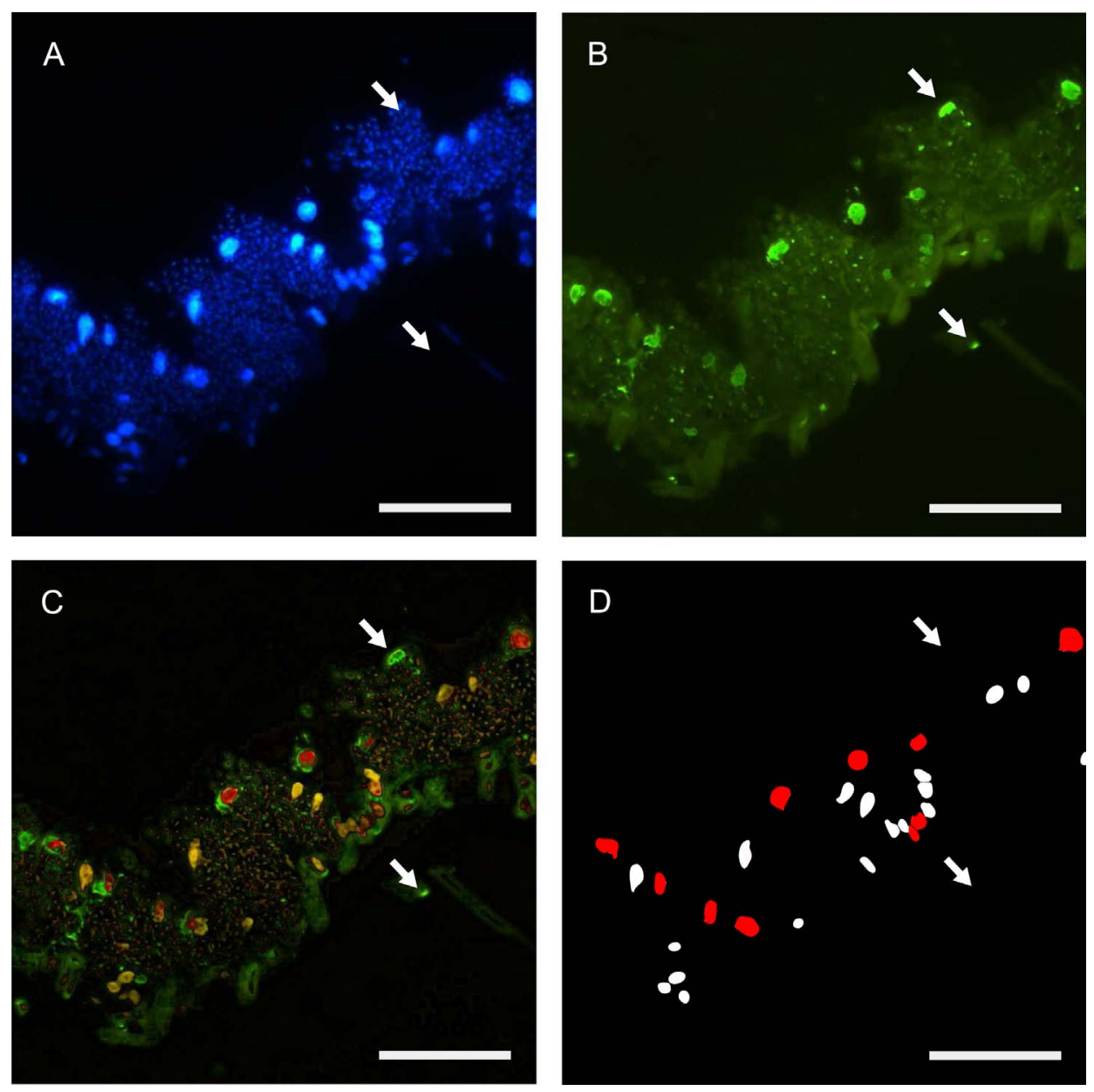

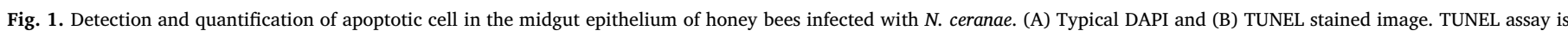

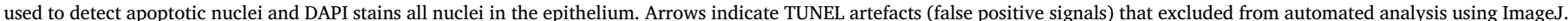

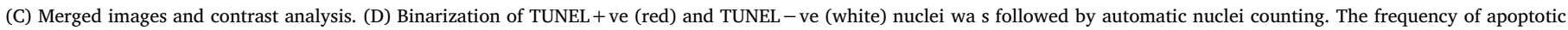
cells as calculated as the numbers of TUNEL + ve relation to all nuclei (DAPI +ve). Scale bars $=50 \mu \mathrm{m}$.

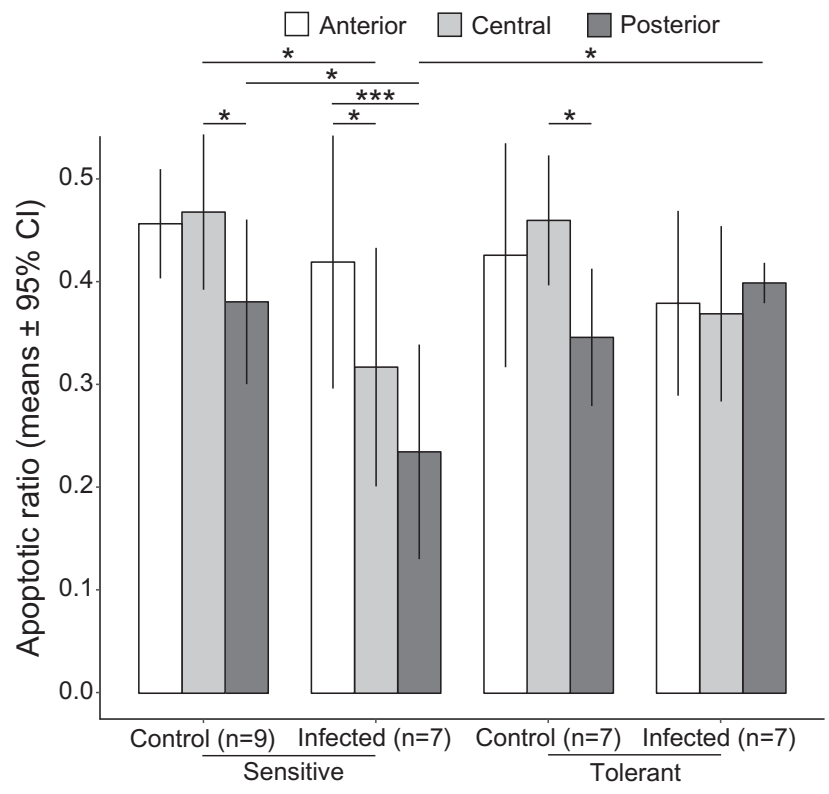

Fig. 2. Differences of apoptosis in the midgut of Nosema sensitive and tolerant honey bees after 6 dpi. Mean ratios of apoptosis $\pm 95 \%$ confidence intervals (CI) for anterior (white), central (light grey) and posterior (dark grey) midgut regions in both control and infected Nosema sensitive and tolerant bees. Significant differences are indicated as "for $\mathrm{p}<0.05$ and ${ }^{* * * *} \mathrm{p}<0.001$.

\section{Acknowledgments}

We thank Claudia Dussaubat and Miriam Widder for their technical support and Theodorou Panagiotis for discussing the statistics. This study was funded by the Deutsche Forschungsgemeinschaft through the priority program "Host-Parasite Coevolution - Rapid Reciprocal Adaptation and its Genetic Basis" (SPP 1399). Grant number MO373/26-2.

\section{Author contributions}

The study was conceived and designed by CK, YLC, PK and RFAM. Experiments were conducted by CK; molecular work and analyses were carried out by CK, OL, TM and RFAM. Manuscript was written by CK, edited by RFAM, and reviewed by YLC, PK, TM, OL.

\section{References}

Antúnez, K., et al., 2009. Immune suppression in the honey bee (Apis mellifera) following infection by Nosema ceranae (Microsporidia). Environ. Microbiol. 11, 2284-2290.

Doublet, V., et al., 2015. Within-host competition among the honey bees pathogens Nosema ceranae and Deformed Wing Virus is asymmetric and to the disadvantage of the virus. J. Invertebr. Pathol. 124, 31-34.

Doublet, V., et al., 2017. Unity in defence: honeybee workers exhibit conserved molecular responses to diverse pathogens. BMC Genomics 18.

Dussaubat, C., et al., 2012. Gut pathology and responses to the microsporidium Nosema ceranae in the honey bee Apis mellifera. PLoS ONE 7.

Fries, I., 1988. Infectivity and multiplication of Nosema apis Z. in the ventriculus of the honey bee. Apidologie 19, 319-328. 
Version définitive du manuscrit publiée dans / Final version of the manuscript published in :

Journal of Invertebrate Pathology (2018), Vol. 154, p. 1-4, DOI: 10.1016/j.jip.2018.03.008

Journal homepage: www.elsevier.com/locate/jip

Fries, I., 2010. Nosema ceranae in European honey bees (Apis mellifera). J. Invertebr. Pathol. 103, S73-S79.

Fries, I., et al., 2013. Standard methods for Nosema research. J. Apicult. Res. 52.

Hatjina, F., et al., 2014. A review of methods used in some European countries for assessing the quality of honey bee queens through their physical characters and the performance of their colonies. J. Apicult. Res. 53, 337-363.

Higes, M., et al., 2013a. Apoptosis in the pathogenesis of Nosema ceranae (Microsporidia: Nosematidae) in honey bees (Apis mellifera). Environ. Microbiol. Rep. 5, 530-536.

Higes, M., et al., 2013b. Nosema ceranae (Microsporidia), a controversial 21st century honey bee pathogen. Environ. Microbiol. Rep. 5, 17-29.

Holt, H.L., et al., 2013. Chronic parasitization by Nosema microsporidia causes global expression changes in core nutritional, metabolic and behavioral pathways in honey bee workers (Apis mellifera). BMC Genomics 14.

Huang, Q., et al., 2012. Survival and immune response of drones of a Nosemosis tolerant honey bee strain towards N. ceranae infections. J. Invertebr. Pathol. 109, 297-302.

Keeling, P., 2009. Five questions about Microsporidia. PLoS Pathog. 5.

Klee, J., et al., 2007. Widespread dispersal of the microsporidian Nosema ceranae, an emergent pathogen of the western honey bee, Apis mellifera. J. Invertebr. Pathol. 96, $1-10$.

Kurze, C., et al., 2016a. Differential proteomics reveals novel insights into Nosema-honey bee interactions. Insect. Biochem. Mol. 79, 42-49.

Kurze, C., et al., 2015. Nosema tolerant honeybees (Apis mellifera) escape parasitic manipulation of apoptosis. PLoS ONE 10, e0140174.
Kurze, C., et al., 2016b. Nosema spp. infections cause no energetic stress in tolerant honeybees. Parasitol. Res. 115, 2381-2388.

Kurze, C., et al., 2016c. Parasite resistance and tolerance in honeybees at the individual and social level. Zoology 119, 290-297.

Lee, J.-H., et al., 2017. Microbiota, gut physiology, and insect immunity. In: Ligoxygakis, P. (Ed.), Adv. Insect Physiol. Academic Press, pp. 111-138 (Chapter 4).

Martin-Hernandez, R., et al., 2017. Microsporidia infection impacts the host cell's cycle and reduces host cell apoptosis. PLOS ONE 12.

Mayack, C., Naug, D., 2009. Energetic stress in the honeybee Apis mellifera from Nosema ceranae infection. J. Invertebr. Pathol. 100, 185-188.

McGee, C.E., et al., 2010. Infection, dissemination, and transmission of a West Nile Virus Green Fluorescent Protein infectious clone by Culex pipiens quinquefasciatus mosquitoes. Vector-Borne Zoonot. 10, 267-274.

Reinbold, C., et al., 2003. Posterior midgut and hindgut are both sites of acquisition of Cucurbit aphid-bome yellows virus in Myzus persicae and Aphis gossypii. J. Gen. Virol. 84, 3473-3484.

Schindelin, J., et al., 2012. Fiji: an open-source platform for biological-image analysis. Nat. Methods 9, 676-682.

Vaidyanathan, R., Scott, T.W., 2006. Apoptosis in mosquito midgut epithelia associated with West Nile virus infection. Apoptosis 11, 1643-1651.

Vidau, C., et al., 2014. Differential proteomic analysis of midguts from Nosema ceranaeinfected honeybees reveals manipulation of key host functions. J. Invertebr. Pathol. 121, 89-96. 